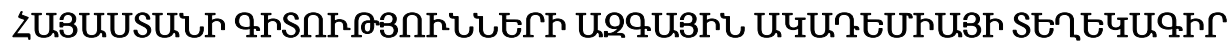 ИЗВЕСТИЯ НАЦИОНАЛЬНОЙ АКАДЕМИИ НАУК АРМЕНИИ
}

\author{
Utipuminlqu \\ 73, №4, 2020 \\ Механика \\ УДК 539.3 \\ Doi- http://doi.org/10.33018/73.4.5

\section{SUPERSONIC FLUTTER OF A COMPRESSED ELONGATED PLATE IN THE PRESENCE OF CONCENTRATED INERTIAL MASSES AND MOMENTS}

\author{
Belubekyan M.V., Martirosyan S.R.
}

Key words Elastic elongated plate, stability, compressive forces, divergence, supersonic panel flutter, concentrated inertial masses and moments

\section{Белубекям М.В., Мартиросян С.P.}

\section{СВЕРХЗВУКОВОЙ ФЛАТТЕР СЖАТОЙ УДЛИНЕННОЙ ПАНЕЛИ ПРИ НАЛИЧИИ СОСРЕДОТОЧЕННЫХ ИНЕРЦИОННЫХ МАСС И МОМЕНТОВ}

Ключевые слова: упругая удлинённая панель, устойчивость, сжимающие силы, сверхзвуковое обтекание, дивергенция панели, локализованная дивергенция, панельный флаттер, сосредоточенные инерционные масса и моменты

В линейной постановке исследуется зависимость видов потери устойчивости невозмущённого состояния рановесия тонкой упругой удлинённой прямоугольной пластинки от характера первоначального напряжённого состояния при наличии сосредоточенных массы и моментов на её свободном крае в предположении, что пластинка сжата в направлении, перпендикулярном скорости обтекающего сверхзвукового потока газа, набегающего на ее свободный край. Найдено аналитическое решение задачи устойчивости возмущённого движения динамической системы «плстинка-поток». Установлено, что при обтекании первоначальное напряжённое состояние, обусловленное сжимающими усилиями, приводит как к существенной дестабилизации, так и к стабилизации состояния невозмущённого равновесия пластинки, в зависимости от параметров системы «пластинка-поток».

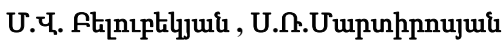

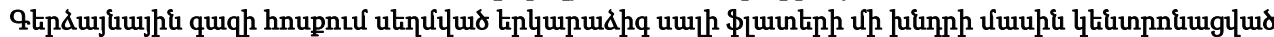

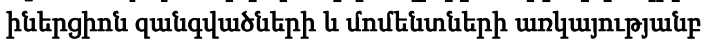

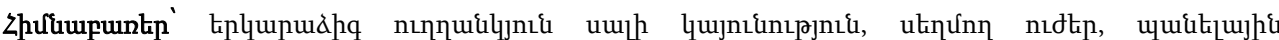

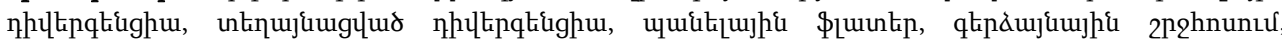
hikinghni quiquluditin la unutiunitip

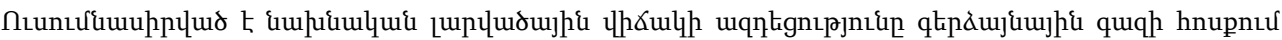

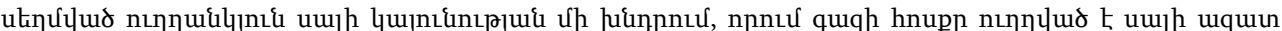

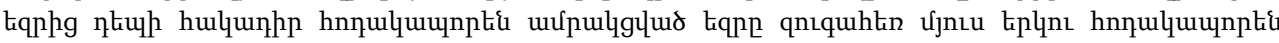

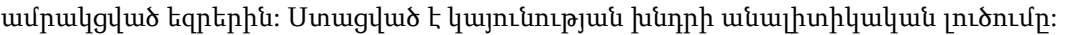

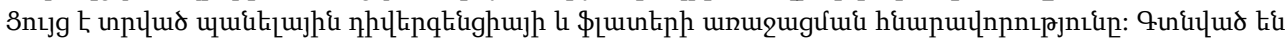

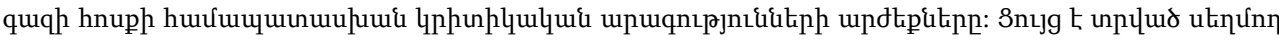

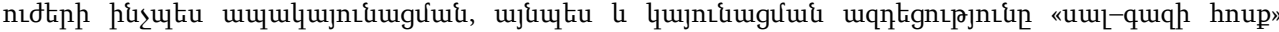

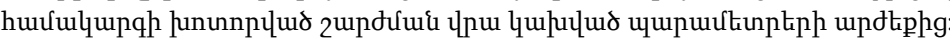


By analyzing, as an example, a thin elastic compressed elongated plate streamlined by supersonic gas flows, we study the influence of the initial stress state of the plate on the stability of the disturbed motion of the dynamic system "plate - flow" under the assumption of presence of concentrated inertial masses and moments on its free edge. We establish the relationship between the characteristics of natural vibrations of the plate and the velocity of the streamlining supersonic gas flow, which enables one to drow some conclusions concerning the stability of disturbed motions of the system depending on the stress factor and the relative thickness of the plate. For various values of the «essential» parameters of the system, we determine the critical velocities of the gas flow leading to the divergence and flutter instability.

Introduction. It is known [1-4] that the specific features of the influence of the initial stress state of the plate on the stability of distributed nonconservative systems are still of great theoretical and practical interest up to the present, since the question concerning elastic stability inevitable arises during disign calculation of any flying mashine to ensure flight safety.

The model problems of the stability of an elastic rectangular plate streamlined by a supersonic gas flow is a mathematical description of the conditions of divergence, localized divergence and panel flutter appearance, excited by air flow.

A huge number of works are devoted to the study of static and dynamic instability of plates and shells, a review of which is mainly contained in monographs and articles [1-10]. However, here, with the exception of A.A. Movchan, approximate solutions are constructed, and an effective estimate of the accuracy of these approximations is not given. A.A. Movchan [4] received the first fundamental theoretical results in such statement at research of a problem about a flutter of the rectangular plate streamlined by supersonic gas flows.

In this article, in a linear statement, we investigate the influence of the initial stress state on the dynamic behavior of the disturbed motion of the "plate-flow" system near the boundaries of the stability region under the following assumptions. A rectangular plate with one free edge and three hinged edges is compressed in a direction perpendicular to the velocity of a supersonic gas flow, incident on its free edge, along which concentrated inertial masses and moments are applied $[2,11]$

Here, in contrast to [1-10], based on the method described in [15], we obtained an analytical solution to the stability problem for the dynamic system "compressed rectangular plate - supersonic gas flow".

The results of the work can be used in the processing of experimental studies of divergence and flutter of the skins of supersonic aircraft, as well as in the study of a wide class of problems of stability of dynamic systems.

\section{Statement of the Problem.}

Consider a thin elastic elongated plate occupying, in a Cartesian coordinate system $O x y z$, a domain $0 \leq x \leq a, 0 \leq y \leq b,-h \leq z \leq h$. We assume that $a b^{-1}<0.2$. The Cartesian coordinate system $O x y z$ is chosen as follows: the $O x$ - and $O y$-axes lie in the plane of the indisturbed plate, and the $\mathrm{Oz}$-axis is perpendicular to the plate and directed to the side of the supersonic gas flow streamlining it on one side in the direction of the $O x$ axis with an undisturbed velocity $V$. It is assumed that the gas flow is plane and potential. 
Suppose that the edge $x=0$ of the plate is free, whereas the edges $x=a, y=0$ and $y=b$ are hinged. The hinges are assumed consider to be perfect. We also assume that concentrated inertial masses $m_{c}$ and rotation moments $I_{c}$ to the free edge $x=0$ are applied $[2,11]$.

Let us assume that initially, even before the flow around, the plate is subject to the action of compressive forces $N_{y}=2 h \sigma_{y}$ uniformly distributed along the edges $y=0$ and $y=b$ of the plate and as a result of heating or some other reason; compressive stresses $\sigma_{y}$ are assumed to be constant throughout the middle surface of the panel, and unchanged with a change a deflection $w=w(x, y, t)$ of the plate $[1,2]$. Under the influence of certain factors, the undisturbed equilibrium state of our plate can be broken down, and it will begin to perform disturbed motion with the deflection $w=w(x, y, t)$.

The deflection $w=w(x, y, t)$ will cause an excess pressure $\Delta p$ onto the upper streamlined surface of the plate from the side of streamlining gas flow, which is taken into account by the approximate formula of the "piston theory" [13,14]: $\Delta p=-a_{0} \rho_{0} V \frac{\partial w}{\partial x}$, where $a_{0}$ is the sound velocity in undisturbed gas medium, and $\rho_{0}$ is the density of undisturbed gas flow. We also assume that the deflections $w=w(x, y, t)$ are small as compared with the plate thickness $2 h$.

Let us find out the conditions under which the loss of stability of the unperturbed state of equilibrium of the dynamic "plate-flow" system is possible, when the bending of the rectangular plate is caused by the corresponding aerodynamic loads $\Delta p$, compressive stresses $\sigma_{y}$ in the middle surface of the plate, concentrated inertial masses $m_{c}$ and rotation moments $I_{c}$. We assume that the compressive forces $\sigma_{y}$ are small as compared to the critical stresses $\left(\sigma_{y}\right)_{c r}$. (tabl. 1) that can produce buckling of the plate in the absence of flow.

Note that in this work, as in work [15], in order to obtain the possibility of an analytical study in the considered problem of the dynamic stability of the "plate - flow" system, the distributed mass of the plate is conditionally replaced by concentrated inertial masses and moments of rotation applied along the free edge of the plate $[2,11]$. Such a replacement does not at all lead to a distortion of the dynamic picture of the phenomenon - loss of system stability; perhaps, up to numerical values of the critical gas flow rates, which can be somewhat overestimated.

Then, under assumption of the validity of the Kirchhoff hypothesis and the "piston theory" $[13,14]$, the small bending vibrations of points of the plate median surface satisfy the differential equation $[2,8]$ :

$D \Delta^{2} w+N_{y} \frac{\partial^{2} w}{\partial y^{2}}+a_{0} \rho_{0} V \frac{\partial w}{\partial x}=0, \quad w=w(x, y, t) ;$

and the corresponding boundary conditions $[2,11]$ : 


$$
\begin{aligned}
& \frac{\partial^{2} w}{\partial x^{2}}+v \frac{\partial^{2} w}{\partial y^{2}}=D^{-1} I_{c} \frac{\partial^{3} w}{\partial x \partial t^{2}}, \frac{\partial}{\partial x}\left(\frac{\partial^{2} w}{\partial x^{2}}+(2-v) \frac{\partial^{2} w}{\partial y^{2}}\right)=-D^{-1} m_{c} \frac{\partial^{2} w}{\partial t^{2}}, x=0 \\
& w=0, \frac{\partial^{2} w}{\partial x^{2}}=0, x=a \\
& w=0, \frac{\partial^{2} w}{\partial y^{2}}=0, \quad y=0 \text { and } y=b
\end{aligned}
$$

Here, $\Delta^{2} w=\Delta(\Delta w), \Delta$ is a Laplace's operator; $D$ is the cylindrical flexural stiffness of our plate; $v$ is Poisson's ratio.

In the parameter space of system (1)-(4), it is required to determine the critical velocity $V_{c r}$. of the supersonic gas flow:

$V_{c r .} \in\left(a_{0} M_{0}, a_{0} M_{2 \cos m .}\right), M_{0}=\sqrt{2}, M_{2 \cos m .} \approx 33.85$;

under the assumption

$\sigma_{y}<\left(\sigma_{y}\right)_{c r .}<\left(\sigma_{y}\right)_{p r .}$;

$M_{0}$ and $M_{2 \cos m .}$ are the boundary values of the Mach number corresponding to the interval of permissible values of supersonic and hypersonic velocities [1]; $\left(\sigma_{y}\right)_{c r}$. is the critical stresses, leading to buckling of the plate in the absence of flow $(V=0)$ [17]; $\left(\sigma_{y}\right)_{p r}$. is the lower limit of fluidity.

In $[15,16]$, an analytical solution was obtained for the stability problem for the dynamic system "plate - flow" in the case in which the influence of the initial stress state of a rectangular plate is not taken into account. And in [17] an analytical solution for the problem of static stability of a panel with loaded edges, both in a streamlined and in the absence of a streamlining are found.

Thus, the analysis of the stability of the disturbed motion of the considered dynamical system "plate - flow" (1) - (4) is reduced to the study of the differential equation (1) with the corresponding boundary conditions (2) - (4) for the deflection under the the assumption (5) and (6).

2. General solution of the problem. For finding the general solution of the problem of stability of the plate (1)-(4), we will reduce it to a problem on eigenvalues for the ordinary differential equation.

We try to find the General solution to the boundary-value problem defined by equation (1) and by the boundary (2) - (4) in the form of harmonic oscillations

$$
w(x, y, t)=\sum_{n=1}^{\infty} C_{n} f_{n}(x) \cdot \sin \left(\mu_{n} y\right) \cdot \exp (\lambda t), \mu_{n}=\pi n b^{-1} ;
$$

where $n$ is the half-waves number in the direction of the plate side $b$.

Then, in accordance with the expression (7), the considered problem of the panel flutter (1)-(4) is reduced to the following boundary value problem on eigenvalues $\lambda$ of nonselfadjoint operator for the ordinary differential equations on the forms of vibrations $f_{n}(x)$ : 
$f_{n}^{I V}(x)-2 \mu_{n}^{2} f_{n}^{I I}(x)+a_{0} \rho_{0} V D^{-1} f_{n}^{I}(x)+\left(\mu_{n}^{4}-N_{y} D^{-1} \mu_{n}^{2}\right) f_{n}(x)=0 ;$

$f_{n}^{I I}-\mu_{n}^{2} v f_{n}(x)=I_{c} D^{-1} \lambda^{2} f_{n}^{I}(x)$,

$f_{n}^{I I I}-\mu_{n}^{2}(2-v) f_{n}^{I}(x)=-m_{c} D^{-1} \lambda^{2} f_{n}(x), \quad x=0$;

$f_{n}(x)=0, \quad f_{n}^{I I}(x)=0, x=a$.

The boundary value problem (8) and (9) consists of investigating the behavior of the eigenvalues $\lambda$ is relation to the velocity $V$ of gas flow. The equilibrium of the plate streamlined by gas flow is stable if all the eigenvalues $\lambda$ lie in the left-hand half-plane of the complex variable. The lowest value of the velocity at which even one of the eigenvalues $\lambda$ crosses into the right-hand half-plane is the critical flutter velocity. Transition into the right-hand half-plane indicates instability of the oscillatory type: there is the panel flutter. The one exception is when transition takes place through the point $\lambda=0$; this case obviously corresponds to the divergence of panel. Here, the coupled interaction of different degrees of freedom is of considerable importance also in the case of panel flutter. Depending on the relation between the parameters, the critical flutter velocity can be either smaller or larger than the critical velocity of divergence.

The particular solution of the differential equation (8) takes the form

$f_{n}(x)=C_{n} \exp \left(\mu_{n} r x\right), \mu_{n}=\pi n b^{-1}$,

$C_{n}$ - are the arbitraries constants, which are not equal to zero simultaneously.

Substituting the solution (10) in the differential equation (6), we obtain the characteristic equation, which is the algebraic equation of the fourth degree

$r^{4}-2 r^{2}+\alpha_{n}^{3} r+\left(1-\beta_{y}^{2}\right)=0, \alpha_{n}^{3}=a_{0} \rho_{0} V D^{-1} \mu_{n}^{-3}, \beta_{y}^{2}=N_{y} D^{-1} \mu_{n}^{-2}$,

which, in accordance with Ferrari's solution, will be represented as [17]:

$\left(r^{2}+\sqrt{2(q+1)} r+q-\sqrt{q^{2}-1+\beta_{y}^{2}}\right) \cdot\left(r^{2}-\sqrt{2(q+1)} r+q+\sqrt{q^{2}-1+\beta_{y}^{2}}\right)=0$.

Here $q$ is the parameter of the velocity $V$ of the gas flow. Parameter $q$ is the real root of the cubic equation

$$
8 \cdot(q+1)\left(q^{2}-1+\beta_{y}^{2}\right)-\alpha_{n}^{6}=0
$$

In this case, the parameter $q$ satisfies the condition [17]:

$q \in\left(q_{0}, \infty\right)$,

$q_{0}=\left(-1+2 \sqrt{\left(4-3 \beta_{y}^{2}\right.}\right) / 3, \beta_{y}^{2} \leq 4 / 3$ and $q_{0}=1, \beta_{y}^{2}>4 / 3$ (tabl. 1).

$\beta_{y}^{2}$ is the compressive stress coefficient, deternined by expressen (11).

Table 1.

\begin{tabular}{|c|c|c|c|c|c|c|c|c|}
\hline$\beta_{y}^{2}$ & 0 & 0.3 & 0.5 & 0.8 & 1.0 & 1.21 & 1.333 & $\geq 1.333$ \\
\hline$q_{0}$ & 1 & 0.840 & 0.721 & 0.510 & 0.338 & 0.072 & -0.333 & 1 \\
\hline
\end{tabular}


In accordance with relation (6), we have

$\beta_{y}^{2}<\left(\beta_{y}^{2}\right)_{c r .},\left(\beta_{y}^{2}\right)_{c r .}=2 h\left(\sigma_{y}\right)_{c r .} D^{-1} \mu_{n}^{-2}=\beta_{y}^{2}(n, \gamma, v)$;

$\gamma$ is a relation of the width $a$ (downstream side) of the elongated plate to its length $b$ :

$\gamma=a b^{-1}, \gamma<0.2$

$\left(\beta_{y}^{2}\right)_{c r}$ is the critical stress coefficient (Table 2) [17].

For all $\gamma \in(0,0.2)$ when $\beta_{y}^{2}<\left(\beta_{y}^{2}\right)_{c r}$. (Table 2) the non-streamlined elongated plate loses its static stability in the form of panel instability.

Table 2.

\begin{tabular}{|c|c|c|c|c|c|}
\hline$\gamma$ & 0.125 & 0.25 & 0.3 & 0.375 & 0.5 \\
\hline 0.0001 & $5.31 \cdot 10^{7}$ & $4.559 \cdot 10^{7}$ & $4.255 \cdot 10^{7}$ & $3.8 \cdot 10^{7}$ & $3.04 \cdot 10^{7}$ \\
\hline 0.001 & $5.31 \cdot 10^{5}$ & $4.559 \cdot 10^{5}$ & $4.255 \cdot 10^{5}$ & $3.8 \cdot 10^{5}$ & $3.04 \cdot 10^{5}$ \\
\hline 0.01 & $5.31 \cdot 10^{3}$ & $4.559 \cdot 10^{3}$ & $4.255 \cdot 10^{3}$ & $3.8 \cdot 10^{5}$ & $3.04 \cdot 10^{3}$ \\
\hline 0.10 & 54.092 & 46.554 & 43.521 & 38.880 & 31.289 \\
\hline 0.30 & 6.822 & 6.029 & 5.695 & 5.180 & 4.272 \\
\hline
\end{tabular}

Note that the stability of non-streamlined rectangular plates under various boundary conditions and different values of the relation sides $\gamma$ of the rectangular plate was considered, in particular, in $[1,3,6,7]$.

In [17], using graphoanalytical research methods, it was shown that in the admissible interval of values of the velocity parameter (15), characteristic equation (11) has two real roots $r_{1}<0, r_{2}<0$, and a pair of complex conjugate roots $r_{3,4} \in W$ with a positive real part, which are easily found as solutions of quadratic equations - factors of relation (12):

$$
\begin{aligned}
& r_{1,2}=-0.5 \sqrt{2(q+1)} \pm \sqrt{\sqrt{q^{2}-1+\beta_{y}^{2}}-0.5(q-1)}, \\
& r_{3,4}=0.5 \sqrt{2(q+1)} \pm i \sqrt{\sqrt{q^{2}-1+\beta_{y}^{2}}+0.5(q-1)} .
\end{aligned}
$$

Moreover, in accordance with condition (15), we have [17]:

$$
\begin{aligned}
& r_{1}<0, r_{2}<0, \text { when } \beta_{y}^{2} \in[0,1), q \in\left(\left(-1+2 \sqrt{4-3 \beta_{y}^{2}}\right) / 3, \infty\right) ; \\
& r_{1}<0, r_{2}>0, \text { when } \beta_{y}^{2} \in(1,4 / 3], q \in\left(\left(-1+2 \sqrt{4-3 \beta_{y}^{2}}\right) / 3, \infty\right) \text { and } \\
& \beta_{y}^{2}>4 / 3, q \in(1, \infty) ; \\
& r_{1}<0, r_{2}=0, \text { when } \beta_{y}^{2}=1, q \in(1 / 3, \infty) .
\end{aligned}
$$

Therefore, the General solution of equation (1), in accordance with the expression (10), will be written as a double row:

$$
w(x, y, t)=\sum_{n=1}^{\infty} \sum_{k=1}^{4} C_{n k} \cdot \exp \left(\mu_{n} r_{k} x+\lambda t\right) \sin \left(\mu_{n} y\right), \mu_{n}=\pi n b^{-1},
$$


$C_{n k}$ is arbitrary constants; $n$ is the number of half-waves along the side $b$ of the plate; $r_{k}$ are the roots of the characteristic equation (11) determined by expressions (18) and (19). In accordance with the notation (11), from relation (13) we determine the explicit form of the dependence of the gas flow velocity $V$ on the parameters of the "plate - flow" system:

$$
V\left(q, n, \gamma, \beta_{y}^{2}\right)=2 \sqrt{2(q+1) \cdot\left(q^{2}-1+\beta_{y}^{2}\right)} \cdot \pi^{3} n^{3} \gamma^{3} D\left(a_{0} \rho_{0} a^{3}\right)^{-1} \text {. }
$$

Taking into account condition (5), it follows that

$V\left(q, n, \gamma, \beta_{y}^{2}\right) \in\left(V\left(q_{0}, n, \gamma, \beta_{y}^{2}\right), a_{0} M_{2 \cos m .}\right) \subseteq\left(a_{0} M_{0}, a_{0} M_{2 \cos m .}\right)$

Hence, taking into account the value of the cylindrical stiffness: $D=\frac{E \cdot(2 h)^{3}}{12 \cdot\left(1-v^{2}\right)}$, it follows that the permissible intervals of the values of the reduced velocity $V\left(q, n, \gamma, \beta_{y}^{2}\right) \cdot D^{-1}\left(a_{0} \rho_{0} a^{3}\right)$ corresponding to the velocity (24) will have the form:

$V\left(q, n, \gamma, \beta_{y}^{2}\right) D^{-1}\left(a_{0} \rho_{0} a^{3}\right) \in\left(V\left(q_{0}, n, \gamma, \beta_{y}^{2}\right) D^{-1}\left(a_{0} \rho_{0} a^{3}\right), a_{0} M_{2 \cos m} \Psi_{1}\right) \subseteq$ $\subseteq\left(a_{0} M_{0} \Psi_{1}, a_{0} M_{2 \cos m .} \Psi_{1}\right)$;

where

$\Psi_{1}=12\left(1-v^{2}\right) a_{0} \rho_{0} E^{-1}\left(2 h a^{-1}\right)^{-3}, M_{0}=\sqrt{2}, M_{2 \cos m .} \approx 33.85$.

The length of the interval (26): $d_{1}\left(v, 2 h a^{-1}\right)=a_{0}\left(M_{2 \cos m .}-M_{0}\right) \Psi_{1}$ is a decreasing function both of the parameter $2 h a^{-1}$ at fixed values of the Poisson's ratio $v$, and of $v$ at fixed values of the relative thickness parameter $2 h a^{-1}$.

3. Sufficient Criteria for the loss of stability of the perturbed motion of the "plateflow" dynamic system (1)-(4).

Substituting the general solution (23) of the differential equation (1) into the boundary conditions (2) - (4), we obtain a homogeneous system of fourth order algebraic equations with respect to arbitrary constants $C_{n k}$. The determinant of this system of equations equated to zero - the characteristic determinant - after simple transformations is reduced to the form of the biquadratic equation:

$\chi_{n} \delta_{n} A_{0} \lambda^{4}+\left(\chi_{n} A_{1}+\delta_{n} A_{2}\right) \lambda^{2}+A_{3}=0$,

where

$\delta_{n}=m_{c} D^{-1} b^{3}(\pi n)^{-3}, \chi_{n}=I_{c} D^{-1} b(\pi n)^{-1}, \delta_{n}>0, \chi_{n}>0$,

are the reduced values, respectively, of the concentrated inertial masses $m_{c}$ and moments of rotation $I_{c}$ applied along the free edge $X=0$ of the plate;

$$
\begin{aligned}
& A_{0}=A_{0}\left(q, n, \gamma, \beta_{y}^{2}\right)=\sqrt{2(q+1)} \cdot\left(1-\exp (-2 \sqrt{2(q+1)} \cdot \pi n \gamma) \cdot B_{1} B_{2}-\right. \\
& -2 B_{2}\left(q+1+\sqrt{q^{2}-1+\beta_{y}^{2}}\right) \cdot \exp (-\sqrt{2(q+1)} \cdot \pi n \gamma) \cdot \operatorname{sh}\left(\pi n \gamma B_{1}\right) \cdot \cos \left(\pi n \gamma B_{2}\right)- \\
& -2 B_{1}\left(q+1-\sqrt{q^{2}-1+\beta_{y}^{2}}\right) \cdot \exp (-\sqrt{2(q+1)} \cdot \pi n \gamma) \cdot \operatorname{ch}\left(\pi n \gamma B_{1}\right) \cdot \sin \left(\pi n \gamma B_{2}\right)
\end{aligned}
$$




$$
\begin{aligned}
& A_{1}=A_{1}\left(q, n, \gamma, \beta_{y}^{2}\right)= \\
& =2(q+1)\left[\left(q-\sqrt{q^{2}-1+\beta_{y}^{2}}\right)+\left(q+\sqrt{q^{2}-1+\beta_{y}^{2}}\right) \exp (-2 \sqrt{2(q+1)} \pi n \gamma] B_{1} B_{2}+\right. \\
& +2 B_{2}\left[\sqrt{2(q+1)\left(q^{2}-1+\beta_{y}^{2}\right)} \cdot\left(q+1+\sqrt{\left(q^{2}-1+\beta_{y}^{2}\right.}\right) \operatorname{sh}\left(\pi n \gamma B_{1}\right)+\right. \\
& \left.+2 B_{1}\left((2 q-1)(q+1)+\beta_{y}^{2}\right) \operatorname{ch}\left(\pi n \gamma B_{1}\right)\right] \cos \left(\pi n \gamma B_{2}\right) \exp (-\sqrt{2(q+1)} \pi n \gamma)+ \\
& +2\left[B_{1} \sqrt{2(q+1)\left(q^{2}-1+\beta_{y}^{2}\right)}\left(q+1-\sqrt{q^{2}-1+\beta_{y}^{2}}\right) \operatorname{ch}\left(\pi n \gamma B_{1}\right)+\right. \\
& \left.+(q+1)\left(q-1+\beta_{y}^{2}\right) \operatorname{sh}\left(\pi n \gamma B_{1}\right)\right] \sin \left(\pi n \gamma B_{2}\right) \cdot \exp (-\sqrt{2(q+1)} \pi n \gamma) ; \\
& A_{2}=A_{2}\left(q, n, \gamma, \beta_{y}^{2}\right)=2(q+1)(1+\exp (-2 \sqrt{2(q+1)} \pi n \gamma)) B_{1} B_{2}- \\
& -4(q+1) B_{1} B_{2} \operatorname{ch}\left(\pi n \gamma B_{1}\right) \cos \left(\pi n \gamma B_{2}\right) \exp (-\sqrt{2(q+1)} \pi n \gamma)+ \\
& +2\left(3\left(q^{2}-1\right)+2 \beta_{y}^{2}\right) \operatorname{sh}\left(\pi n \gamma B_{1}\right) \sin \left(\pi n \gamma B_{2}\right) \exp (-\sqrt{2(q+1)} \pi n \gamma) ; \\
& A_{3}=A_{3}\left(q, n, \gamma, v, \beta_{y}^{2}\right)= \\
& =\sqrt{2(q+1)}\left\{\left(q+1-\sqrt{q^{2}-1+\beta_{y}^{2}}\right)^{2}-2(q+1) \cdot v-(1-v)^{2}\right\} B_{1} B_{2}- \\
& -\sqrt{2(q+1)}\left\{\left(q+1+\sqrt{q^{2}-1+\beta_{y}^{2}}\right)^{2}-2(q+1) \cdot v-(1-v)^{2}\right\} B_{1} B_{2} \cdot \\
& \exp (-2 \sqrt{2(q+1)} \pi n \gamma)+2\left\{\left[\left(4 q^{2}+2 q-1\right) \sqrt{q^{2}-1+\beta_{y}^{2}}-\left(2 q^{2}-4 q+1\right)(\mathrm{q}+1)-\right.\right. \\
& -\left(q-1-\sqrt{q^{2}-1+\beta_{y}^{2}}\right) \cdot \beta_{y}^{2}-2\left((2 q-1)(\mathrm{q}+1)-q \sqrt{q^{2}-1+\beta_{y}^{2}}+\beta_{y}^{2}\right) v+ \\
& \left.+\left(\mathrm{q}+1+\sqrt{q^{2}-1+\beta_{y}^{2}}\right) v^{2}\right] \operatorname{sh}\left(\pi n \gamma B_{1}\right)+2 \sqrt{2(q+1)\left(q^{2}-1+\beta_{y}^{2}\right)} \cdot(q+1) B_{1} . \\
& \left.\cdot \operatorname{ch}\left(\pi n \gamma B_{1}\right)\right\} B_{2} \cos \left(\pi n \gamma B_{2}\right) \exp (-\sqrt{2(q+1)} \pi n \gamma)+ \\
& +2\left\{-B_{1}\left[\left(4 q^{2}+2 q-1\right) \sqrt{q^{2}-1+\beta_{y}^{2}}+\left(2 q^{2}-4 q+1\right)(\mathrm{q}+1)+\right.\right. \\
& +\left(q-1+\sqrt{q^{2}-1+\beta_{y}^{2}}\right) \beta_{y}^{2}+2\left((2 q-1)(\mathrm{q}+1)+q \sqrt{q^{2}-1+\beta_{y}^{2}}+\beta_{y}^{2}\right) v- \\
& \left.-\left(q+1-\sqrt{q^{2}-1+\beta_{y}^{2}}\right) v^{2}\right] \cdot \operatorname{ch}\left(\pi n \gamma B_{1}\right)-\sqrt{2(q+1)} \cdot\left(3\left(q^{2}-1\right)+2 \beta_{y}^{2}\right) \text {. } \\
& \left.\sqrt{q^{2}-1+\beta_{y}^{2}} \cdot \operatorname{sh}\left(\pi n \gamma B_{1}\right)\right\} \cdot \sin \left(\pi n \gamma B_{2}\right) \exp (-\sqrt{2(q+1)} \pi n \gamma)=0 \text {; } \\
& \text { where } \\
& B_{1}=\sqrt{\sqrt{q^{2}-1+\beta_{y}^{2}}-0.5(q-1)}, \quad B_{2}=\sqrt{\sqrt{q^{2}-1+\beta_{y}^{2}}+0.5(q-1)} .
\end{aligned}
$$


For all admissible values of the velocity parameter $q \in\left(q_{0}, \infty\right)$ (Table 1), stress coefficient $\beta_{y}^{2}<\left(\beta_{y}^{2}\right)_{c r}$. (Table 2), parameters $n \geq 1$ and $\gamma \in(0, \infty)$ it is obviously that $B_{1}=B_{1}\left(q, \beta_{y}^{2}\right)>0$ and $B_{2}=B_{2}\left(q, \beta_{y}^{2}\right)>0$, whence the validity of the inequalities follows:

$A_{0}=A_{0}\left(q, n, \gamma, \beta_{y}^{2}\right)>0, A_{2}=A_{2}\left(q, n, \gamma, \beta_{y}^{2}\right)>0$.

Introducing the notation

$k_{n}=\chi_{n} \cdot \delta_{n}^{-1}$,

characteristic determinant (28), in accordance with conditions (29) and (35), can be rewritten in the form

$\lambda^{4}+\left(k_{n} A_{1}+A_{2}\right) \chi_{n}^{-1} A_{0}^{-1} \lambda^{2}+\chi_{n}^{-1} \delta_{n}^{-1} A_{0}^{-1} A_{3}=0, \delta_{n}>0, \chi_{n}>0, k_{n}>0$.

The analysis of the stability of the unperturbed equilibrium state of the dynamic system "plate-flow" (1) - (4) is reduced to the study of the behavior of the roots $\lambda_{k}$ of the characteristic determinant (37), which determine the proper motions of the system in the space of «essential» parameters $\mathfrak{I}=\left\{q(V), n, \gamma, v, \beta_{y}^{2}, k_{n}\right\}$. There are have the most significant effect on the dynamic behavior of the system. The values of the other parameters of the system are taken to be fixed.

\section{Partitioning the parameter space $\mathfrak{I}$ of the system «plate-flow» into domains of stability and instability.}

Let us introduce into consideration in the parameter space $\mathfrak{I}$ the domain of stability $\mathfrak{I}_{0}$ and the domain $\mathfrak{I}_{1}, \mathfrak{I}_{2}, \mathfrak{I}_{3}$ of instability. In the domain $\mathfrak{I}_{0}$ all roots $\lambda_{k}$ of the characteristic equation (37) are in the left-hand side of the complex plane. The domain $\mathfrak{I}_{1}, \mathfrak{I}_{2}, \mathfrak{I}_{3}$, respectively, is one positive root among the roots $\lambda_{k}$, or there are two positive roots, or there is a pair of complex conjugates roots with positive real part. It is clear that the perturbed motion of the system is stable for values of parameters from the domain $\mathfrak{I}_{0}$ and unstable for values of parameters from $\mathfrak{I}_{1}, \mathfrak{I}_{2}, \mathfrak{I}_{3}$ domains.

The system "plate-flow" in domains $\mathfrak{I}_{1}$ and $\mathfrak{I}_{2}$ loses its stability in the form of panel divergence, and in domain $\mathfrak{I}_{3}$ in the form of a panel flutter. The panel divergence in domain $\mathfrak{I}_{2}$ is more pronounced.

The stability domain $\mathfrak{I}_{0} \in \mathfrak{I}$ is determined by the relations:

$k_{n} A_{1}+A_{2}>0, A_{3}>0, \Delta>0$.

And the instability domains $\mathfrak{I}_{1}, \mathfrak{I}_{2}, \mathfrak{I}_{3}$ will be determined, respectively, by the relations:

$A_{3}<0, \Delta>0 ; \Delta>0, k_{n} A_{1}+A_{2}<0$ and $A_{3}>0, \Delta<0$.

Here, $\Delta$ is the discriminant of the characteristic determinate (37):

$\Delta=\Delta\left(n, \gamma, \nu, \beta_{y}^{2}, k_{n}\right)=\left(k_{n} A_{1}+A_{2}\right)^{2}-4 k_{n} A_{0} A_{3}$. 
It is obviously, that under the conditions (38) the equation (37) has two pairs of purely imaginary roots $\lambda_{1,2}= \pm i \omega_{1}, \lambda_{3,4}= \pm i \omega_{2}$. The rectangular plate in this case performs harmonic oscillations about the undisturbed equilibrium state.

The boundaries of the stability domain $\mathfrak{I}_{0} \in \mathfrak{I}$ under the condition $k_{n} A_{1}+A_{2}>0$ are hypersurfaces $A_{3}=0$ and $\Delta=0[12,15]$. The characteristic equation (37) on the hypersurfaces $A_{3}=0$ and $\Delta=0$ has one zero root $\lambda_{0}=0$ of multiplicity 2 and a pair $\lambda_{1,2}= \pm i \omega$ of purely imaginary roots respectively.

On the boundary $\Delta>0, \quad k_{n} A_{1}+A_{2}>0, A_{3}=0$ of the stability domain $\mathfrak{I}_{0}$ the disturbed motion of the "plate-flow" system loses its static stability in the form of the panel divergence at flow velocities $V \geq V_{\text {cr.div. }}$ under the condition $\beta_{y}^{2}<\left(\beta_{y}^{2}\right)_{c r}$ (Table 2). Substituting the first root $q_{\text {cr.div }}$ of the equation (33) in the formula (24), we obtain the reduced critical divergence velocity $V_{c r \cdot d i v} D^{-1}\left(a_{0} \rho_{0} a^{3}\right)$.

On the boundary $\Delta=0, k_{n} A_{1}+A_{2}>0, A_{3}>0$ of the stability domain $\mathfrak{I}_{0}$, as well as on the boundary $\Delta=0, k_{n} A_{1}+A_{2}<0, A_{3}>0$ the static instability domain $\mathfrak{J}_{2}$ the disturbed motion of the system «plate-flow» loses its the dynamic stability: the plate makes the flutter oscillations. The critical velocities $V_{\text {cr.fl. }}$ of the gas flow that corresponds to the first root $q_{c r . f l}$ of the equation (40): calculated according to the formula (24)., delimits or When the velocities $V \geq V_{\text {cr.fl. }}$ of the gas flow is "soft" ("smooth") transition to oscillations of increasing amplitude (i.e. to the flutter oscillations). In the first case, the harmonic oscillations are gradually moving in the flutter oscillations, while in the second case, the transition in the flutter oscillations occurs along with a monotonic "buckling" the surface of the plate.

From the equations (33), (40) we can drow certain qualitative and quantitative conclusions concerning the influence of a number of parameters on the stability of the plane form of the plate.

Thus, the critical velocities of divergence instability $V_{c r \text {.div }}$ and of flutter instability $V_{c r \text {.fl. }}$ of the disturbed motion of the rectangular plate, corresponding, to the roots $q_{c r \text {.div. }}$ and $q_{c r . f l}$. of the equations (33) and (40) respectively, are determined by the formula (16) with sufficient accuracy. At $V \geq V_{c r . d i v}$ and $V \geq V_{c r . f l}$. velocities there is the "soft" loss of stability of disturbed motion of the system «plate-flow» in the forms of divergence and panel flutter forms respectively.

5. Numerical results. Using the graphic-analytical and numerical methods of analysis, we constructed the family of curves $\left\{q\left(n, \gamma, \nu, \beta_{y}^{2}, k_{n}\right)\right\}$ parameterized properly in the multiparameter space $\mathfrak{I}$ of our problem. 
Numerical calculations carried out for various values of the number of half-waves $n$ have shown that for fixed values of the remaining parameters of the system, the value $n=1$ corresponds to the smallest values of the critical velocities of divergence and flutter.

Tables 3-10 present the numerical results of solving the original problem of stability (1) (4), characterizing the most representative cases of the dependence of the reduced critical velocities of divergence and flutter on moderate values of the «essential» parameters of the problem as applied to the interval of super- and hypersonic velocities (26) for the elongated plate $(\gamma<0.2)$.

For a clear illustration of the dynamics of the state of the "plate - flow" system in the space $\mathfrak{I}$, we will compose chains of transitions from domain $\mathfrak{I}_{l}$ to domain $\mathfrak{I}_{k}$, based on the analysis of numerical results.

Chains of transitions have the following representation:

$$
\begin{aligned}
& \mathfrak{I}_{1} \stackrel{V_{0}}{\longrightarrow} \mathfrak{I}_{0} \stackrel{V_{\text {crdiv }}}{\longrightarrow} \mathfrak{I}_{1}, k_{n}=0 ; \\
& \mathfrak{I}_{1} \stackrel{V_{2}}{\longrightarrow} \mathfrak{I}_{2} \stackrel{V_{\text {crfl }}}{\longrightarrow} \mathfrak{I}_{3} \stackrel{V_{0}^{*}}{\longrightarrow} \mathfrak{I}_{0} \stackrel{V_{\text {crdiv }}}{\longrightarrow} \mathfrak{I}_{1}, k_{n}>0 .
\end{aligned}
$$

Hence, it can be seen that at gas flow velocities $V \geq a_{0} M_{0}=a_{0} \sqrt{2}$ the "elongated plate - flow" system is statically unstable: there is a panel divergence. However, in accordance with chain (5.1), when $k_{n}=0$ the "plate - flow" system becomes stable at velocities gas flow $V \geq V_{0}$, after which it loses stability again at velocities $V \geq V_{c r \text { div }}$ in the form of panel divergence. In the case, the flutter is absent.

According to representation (5.2), in the case $k_{n}>0$, the system at the gas flow velocities $V \geq V_{2}$ transitions into the domain of the panel divergence $\mathfrak{I}_{2}$, from where, at $V \geq V_{\text {cr.fl. }}$, it transitions into the domain of the flutter oscillations $\mathfrak{I}_{3}$.

If, after that, the plate material does not destruction, then with a further increase in the gas flow velocity, the disturbed motion of the system at velocities $V \geq V_{0}^{*}$ becomes stable, after which, at gas flow velocities $V \geq V_{c r . d i v}$, it loses static stability.

Table 3.

\begin{tabular}{|l|c|c|c|c|c|c|}
\hline \multicolumn{1}{|c|}{$\beta_{y}^{2}$} & 0 & 1 & 5 & 10 & 20 & 30 \\
$\gamma=0.1$ & & & & & & \\
\hline$V_{0} D^{-1}\left(a_{0} \rho_{0} a^{3}\right)$ & 76.764 & 77.753 & 80.246 & 83.608 & 90.199 & 96.815 \\
$\left(k_{1}=0\right) ;$ & 76.823 & 77.867 & 80.394 & 83.713 & 90.326 & 96.954 \\
$V_{2} D^{-1}\left(a_{0} \rho_{0} a^{3}\right)$ & 76.893 & 77.942 & 80.501 & 83758 & 90.464 & 97.086 \\
& 77.003 & 78.112 & 80.618 & 83.937 & 90.612 & 97.257 \\
$V_{\text {cr.div. }} D^{-1}\left(a_{0} \rho_{0} a^{3}\right)$ & 485.155 & 78.195 & 80.756 & 84.125 & 90.730 & 97.497 \\
$\left(k_{1} \geq 0\right)$ & 484.509 & 486.720 & 490.721 & 497.367 & 509.391 & 521.747 \\
& 484.045 & 485.673 & 489.554 & 495.962 & 507.974 & 520.320 \\
& 483.580 & 484.512 & 488.982 & 495.611 & 507.503 & 519.844 \\
& 482.188 & 483.351 & 487.843 & 494.862 & 506.795 & 518.893 \\
\end{tabular}


Note that the limiting velocity $V_{2}$ at $k_{n}>0$ is exactly equal to the velocity $V_{0}$ at $k_{n}=0$. This means that, in this case, even an arbitrarily small value of the turning moment $I_{c}$ leads to significant destabilization of the state of the system.

According to representation (5.2), it is clear that $V_{\text {cr.fl. }}<V_{0}^{*}<V_{\text {cr.div. }}$ at all $\gamma<0.2$ and $k_{n}>0$.

Table 4.

\begin{tabular}{|c|c|c|c|c|c|c|c|c|}
\hline $\begin{array}{c}k_{1} \\
\beta_{y}^{2}\end{array}$ & 0.1 & 0.3 & 0.5 & 1 & 5 & 10 & 20 & 200 \\
\hline 0 & 264.95 & 222.49 & 208.09 & 193.75 & 175.56 & 171.19 & 168.32 & 163.53 \\
\hline 30 & 242.12 & 208.65 & 197.46 & 186.43 & 172.26 & 169.14 & 166.86 & 163.21 \\
\hline
\end{tabular}

Table 5.

\begin{tabular}{|r|c|c|c|c|c|c|}
\hline$\beta_{y}^{2}$ & 0 & 1 & 5 & 10 & 20 & 30 \\
\hline 0.1 & 92.615 & 93.206 & 95.514 & 98.293 & 103.682 & 108.577 \\
\hline 0.3 & 114.863 & 115.299 & 117.047 & 118.516 & 122.940 & 125.931 \\
\hline 0.5 & 123.892 & 124.192 & 125.834 & 127.338 & 130.364 & 132.808 \\
\hline 0.8 & 131.087 & 131266 & 131.808 & 133.336 & 136.369 & 138.734 \\
\hline 1.0 & 133.953 & 134.205 & 134.831 & 136.363 & 138.696 & 140.575 \\
\hline 5.0 & 148.690 & 148.928 & 149.021 & 149.825 & 151.120 & 152.467 \\
\hline 10.0 & 152.545 & 152.837 & 153.135 & 153.452 & 154.280 & 154.952 \\
\hline 20.0 & 155.240 & 155.323 & 155.496 & 155.833 & 156.506 & 157.021 \\
\hline 200.0 & 159.255 & 159.324 & 159.393 & 159.523 & 159.770 & 159.943 \\
\hline
\end{tabular}

Tables 3-5 and 6-8 give the values of the reduced critical velocities, $V_{c r d i v} D^{-1}\left(a_{0} \rho_{0} a^{3}\right)$ and $V_{0} D^{-1}\left(a_{0} \rho_{0} a^{3}\right)$ (Tables 3 and 6), (Tables 4 and 7 ), $V_{0}^{*} D^{-1}\left(a_{0} \rho_{0} a^{3}\right)$ and $V_{\text {cr.fl. }} D^{-1}\left(a_{0} \rho_{0} a^{3}\right)$ (Tables 5 and 8 ) for $\gamma=0.1$ and $\gamma=0.01$, respectively, in relation to the interval (5) at condition (26) when $n=1$.

The reduced critical divergence velocity of the panel $V_{c r d i v} D^{-1}\left(a_{0} \rho_{0} a^{3}\right)$ does not depend on $k_{n}=\chi_{n} \delta_{n}^{-1}$, but depends only on the Poisson's ratio $v$, parameter $\gamma$ and stress koefficient $\beta_{y}^{2}$ : less in plates made of materials with a high Poisson's ratio $v$, decreases with decreasing $\gamma$, and with increasing $\beta_{y}^{2}$ the critical divergence velocity increases (Tables 3 and 6). The values $V_{c r d i} D^{-1}\left(a_{0} \rho_{0} a^{3}\right)$ at the same $\beta_{y}^{2}$ correspond, respectively, to values $v$ of $0.125 ; 0.25 ; 0.3 ; 0.375$ and 0.5 (Table 3). Note that with a decrease $\gamma$, 
starting approximately from $\gamma=0.01$, the influence of the parameters $\nu$ and $\beta_{y}^{2}$ on the critical velocity $V_{\text {crdiv }} \cdot D^{-1}\left(a_{0} \rho_{0} a^{3}\right)$ becomes imperceptibly small.

Table 6.

\begin{tabular}{|c|c|c|c|c|c|c|}
\hline$\gamma=0.01$ & 0 & 100 & 500 & 1000 & 2000 & 3000 \\
\hline $\begin{array}{l}V_{0} D^{-1}\left(a_{0} \rho_{0} a^{3}\right) \\
\left(k_{1}=0\right) \\
V_{2} D^{-1}\left(a_{0} \rho_{0} a^{3}\right) \\
\left(k_{1}>0\right)\end{array}$ & 75.764 & 77.014 & 78.914 & 82.172 & 88.883 & 95.159 \\
\hline $\begin{array}{l}V_{\text {cr.div. }} D^{-1}\left(a_{0} \rho_{0} a^{3}\right. \\
\left(k_{1} \geq 0\right)\end{array}$ & 484.898 & 487.134 & 490.721 & 494.225 & 508.322 & 520.171 \\
\hline
\end{tabular}

Table 7 .

\begin{tabular}{|l|c|c|c|c|c|c|c|c|}
\hline $\begin{array}{c}k_{1} \\
\beta_{y}^{2}\end{array}$ & 0.1 & 0.3 & 0.5 & 1 & 5 & 10 & 20 & 200 \\
\hline 0 & 170.62 & 166.53 & 165.23 & 163.90 & 162.22 & 161.84 & 161.51 & 161.03 \\
\hline 3000 & 168.49 & 165.06 & 164.10 & 163.21 & 161.92 & 161.59 & 161.35 & 160.99 \\
\hline
\end{tabular}

The reduced critical stability velocity $V_{0} D^{-1}\left(a_{0} \rho_{0} a^{3}\right)$ at $k_{n}=0$, as well as the reduced divergence velocity of the panel $V_{2} D^{-1}\left(a_{0} \rho_{0} a^{3}\right)$ at $k_{n}>0$, depends on the parameters $\gamma, \beta_{y}^{2}$ and $v$ : decrease with decreasing $\gamma$, increase with increasing $\beta_{y}^{2}$, and less in plates made of materials with a lower Poisson's ratio $v$ (Tables 3 and 6).

The reduced critical stability velocity $V_{0}^{*} D^{-1}\left(a_{0} \rho_{0} a^{3}\right)$ (Tables 4 and 7 ) decreases with decreasing $\gamma$, decreases with increasing parameters $\beta_{y}^{2}$ and $k_{n}$, and, more in plates made of materials with a large Poisson's ratio $v$. The reduced critical flutter velocity $V_{c r . f l .} D^{-1}\left(a_{0} \rho_{0} a^{3}\right)$ increases with decreasing $\gamma$, increases with increasing parameters $\beta_{y}^{2}$ and $k_{n}$, and more in plates made of materials with a high Poisson's ratio $v$ (Tables 5 and 8$)$. In this case, in contrast to the reduced critical velocity of the panel divergence in the panel $V_{c r d i v} \cdot D^{-1}\left(a_{0} \rho_{0} a^{3}\right)$, the effect of Poisson's ratio $v$ on the reduced critical velocities of stability and flutter is imperceptibly small.

70 
Table 8.

\begin{tabular}{|c|c|c|c|c|c|c|}
\hline$\beta_{y}^{2}$ & 0 & 100 & 500 & 1000 & 2000 & 3000 \\
\hline 0.1 & 151.314 & 151.645 & 152.103 & 152.260 & 152.893 & 153.684 \\
\hline 0.3 & 155.271 & 155.367 & 155.590 & 155.748 & 156.385 & 156.624 \\
\hline 0.5 & 156.545 & 156.689 & 156.864 & 157.023 & 157.342 & 157.582 \\
\hline 0.8 & 157.502 & 157.568 & 157.662 & 157.821 & 157.982 & 158.301 \\
\hline 1.0 & 157.820 & 157.902 & 157.982 & 158.094 & 158.301 & 158.542 \\
\hline 5.0 & 159.504 & 159.541 & 159.583 & 159.664 & 159.744 & 159.824 \\
\hline 10.0 & 159.905 & 159.922 & 159.936 & 159.965 & 160.017 & 160.049 \\
\hline 20.0 & 160.178 & 160.189 & 160.209 & 160.225 & 160.242 & 160.306 \\
\hline 200.0 & 160.644 & 160.648 & 160.652 & 160.658 & 160.676 & 160.692 \\
\hline
\end{tabular}

As follows from the results of numerical analysis, as the parameter $\gamma$ decreases, the dependence of the reduced critical velocities of stability, divergence and flutter of the "plate-flow" system on the parameters $\gamma, \beta_{y}^{2}<\left(\beta_{y}^{2}\right)_{c r}$ and Poisson's ratio $v$ becomes imperceptibly small. Therefore, at $\gamma \ll 1$, the reduced critical divergence velocity can be considered constant, and the critical stability and flutter velocities depend only on the coefficient $k_{1}>0$, which is in full agreement with the results of [15].

It is easy to find the minimum relative thickness $\left(2 h a^{-1}\right)_{\min }$ [2] of an elongated rectangular plate $(\gamma<0.2)$ at which the perturbed motion of the dynamic "plate - flow" system at gas flow velocities near $a_{0} \sqrt{2}$ is stable.

Indeed, assuming that $\left(V_{0}\right)_{\max } \leq a_{0} \sqrt{2}$ for all $k_{n} \geq 0$, from the definition of the reduced gas flow rate and the expression of the cylindrical stiffness, we obtain the formula for calculating the minimum relative thickness of the plate:

$\left(2 h a^{-1}\right)_{\min }^{3}=\frac{a_{0} \sqrt{2}}{\left(V_{0 \max } D^{-1}\left(a_{0} \rho_{0} a^{3}\right)\right)} \cdot\left(12\left(1-v^{2}\right) a_{0} \rho_{0} E^{-1}\right)$

Substituting into expression (43) the corresponding values $\left(V_{0}\right)_{\max } D^{-1}\left(a_{0} \rho_{0} a^{3}\right)$ (tabl.

3 and 6) and $\left(V_{0}\right)_{\max }^{*} D^{-1}\left(a_{0} \rho_{0} a^{3}\right)$ (tabl. 4 and 7), we obtain the following values $\left(2 h a^{-1}\right)_{\min }$ for the steel plate:

$\left(2 h a^{-1}\right)_{\min } \approx 0.0045$ for $k_{n}=0$ and for all $0<\gamma<0.2$;

$\left(2 h a^{-1}\right)_{\min } \approx 0.00345$ for $k_{n}>0$ and $\gamma=0.1$;

$\left(2 h a^{-1}\right)_{\min } \approx 0.00398$ for $k_{n}>0$ and $0<\gamma \leq 0.01$. 
Then, for all $\left(2 h a^{-1}\right)<\left(2 h a^{-1}\right)_{\min }$ and $\gamma<0.2$, the system becomes stable near $a_{0} \sqrt{2}$, and the transition chains will have the following representation:

for $\gamma=0.1$

$\mathfrak{I}_{0} \stackrel{V_{\text {crdiv }}}{\longrightarrow} \mathfrak{I}_{11} \rightarrow \mathfrak{I}_{0} \rightarrow \mathfrak{I}_{11}\left(k_{n}=0\right)$;

$\mathfrak{I}_{0} \stackrel{V_{\text {cr.div. }}}{\longrightarrow} \mathfrak{I}_{11} \rightarrow \mathfrak{I}_{12} \rightarrow \mathfrak{I}_{11} \rightarrow \mathfrak{I}_{0} \stackrel{\tilde{V}_{\text {cr.fl. }}}{\longrightarrow} \mathfrak{I}_{3} \rightarrow \mathfrak{I}_{0} \rightarrow \mathfrak{I}_{11}\left(0<k_{n} \leq 0.1\right) ;$

$\mathfrak{I}_{0} \stackrel{V_{\text {cr.div. }}}{\longrightarrow} \mathfrak{I}_{11} \rightarrow \mathfrak{I}_{12} \rightarrow \mathfrak{I}_{2} \stackrel{\tilde{V}_{\text {cr.fl. }}}{\longrightarrow} \mathfrak{I}_{3} \rightarrow \mathfrak{I}_{0} \rightarrow \mathfrak{I}_{11}\left(k_{n}>0.1\right) ;$

for $\gamma=0.01$

$\mathfrak{I}_{0} \stackrel{V_{c r \text { div. }}}{\longrightarrow} \mathfrak{I}_{11} \rightarrow \mathfrak{I}_{0} \rightarrow \mathfrak{I}_{11}\left(k_{n}=0\right)$;

$\mathfrak{I}_{0} \stackrel{V_{\text {cr.div. }}}{\longrightarrow} \mathfrak{I}_{11} \rightarrow \mathfrak{I}_{12} \rightarrow \mathfrak{I}_{2} \stackrel{\tilde{V}_{\text {cr.fl. }}}{\longrightarrow} \mathfrak{I}_{3} \rightarrow \mathfrak{I}_{0} \rightarrow \mathfrak{I}_{11}\left(k_{n}>0\right)$.

Moreover, it is obviously for all $\gamma<0.2$ that $V_{0}<V_{\text {cr.div. }}$ at $k_{n}=0$ and $V_{0}<V_{\text {cr.div. }}<\tilde{V}_{\text {cr.fl. }}$ at $k_{n}>0$.

For $\gamma=0.1$ and $\gamma=0.01$ the values $V_{c r . d i v} D^{-1}\left(a_{0} \rho_{0} a^{3}\right)$ and $\tilde{V}_{c r . f l} D^{-1}\left(a_{0} \rho_{0} a^{3}\right)$ are given in tables 3,9 and tables 6,10 respectively. As it turned out, in this case, the reduced critical divergence velocity do not depend on the relative plate thickness, in contrast to the reduced critical flutter velocity, which grow with decreasing relative plate thickness.

Table 9.

\begin{tabular}{|r|c|c|c|c|c|c|}
\hline \multicolumn{1}{|r|}{$\beta_{y}^{2}$} & 0 & 1 & 5 & 10 & 20 & 30 \\
\hline 0.1 & 1544.185 & 1547.963 & 1557.448 & 1567.503 & 1594.023 & 1621.745 \\
\hline 0.3 & 1573.336 & 1575.717 & 1581.917 & 1588.723 & 1604.249 & 1630.425 \\
\hline 0.5 & 1628.700 & 1631.824 & 1636.715 & 1644.566 & 1658.251 & 1671.874 \\
\hline 0.8 & 1688.219 & 1688.983 & 1694.037 & 1700.213 & 1712.586 & 1721.892 \\
\hline 1.0 & 1714.703 & 1716.213 & 1720.905 & 1726.572 & 1738.815 & 1749.518 \\
\hline 5.0 & 1874.636 & 1874.861 & 1878.214 & 1881.316 & 1887.998 & 1894.761 \\
\hline 10.0 & 1920.410 & 1920.679 & 1922.902 & 1925.923 & 1930.898 & 1936.057 \\
\hline 20.0 & 1954.703 & 1955.443 & 1955.992 & 1959.131 & 1962.845 & 1966.137 \\
\hline 200.0 & 2014.922 & 2015.007 & 2015.669 & 2017.064 & 2018.845 & 2020.490 \\
\hline
\end{tabular}

Table 10.

\begin{tabular}{|l|c|c|c|c|c|c|c|c|}
\hline $\begin{array}{c}k_{1} \\
\beta_{y}^{2}\end{array}$ & 0.1 & 0.3 & 0.5 & 1 & 5 & 10 & 20 & 200 \\
\hline 0 & 1907.6 & 1967.7 & 1981.4 & 2002.2 & 2024.3 & 2029.9 & 2032.9 & 2040.4 \\
\hline 3000 & 1925.9 & 1975.8 & 1984.3 & 2005.6 & 2026.1 & 2030.4 & 2033.6 & 2040.8 \\
\hline
\end{tabular}


From a comparison of the data in tables 5, 9 for $\gamma=0.1$ and data in tables 8, 10 for $\gamma=0.01$, it follows that $\tilde{V}_{c r . f l .} D^{-1}\left(a_{0} \rho_{0} a^{3}\right)>V_{c r . f l .} D^{-1}\left(a_{0} \rho_{0} a^{3}\right)$ an order of magnitude or more.

Thus, in the case of elongated plates $(\gamma<0.2)$, the reduced critical velocities of divergence and flutter are increasing functions of the stress coefficient $\beta_{y}^{2}$ characterizing the initial stressed state of the plate: the initial stressed state leads to stabilization of the disturbed motion of the dynamic "plate - flow" system, in comparison with the behavior of the dynamic system with unloaded panel [15]. However, with decreasing $\gamma$, when $\gamma \ll 1$, the influence of the initial stress state on the stability of the disturbed motion of the system becomes imperceptibly small.

\section{Conclusitions}

An analytical solution to the problem of stability of the disturbed motion of a dynamic system "plate - flow" is found under the assumption that the edges of a elongated plate, parallel to the gas flow velocity, are loaded even before the flow streamlined by uniformly distributed compressive forces.

Explicit expressions of sufficient criteria for the loss of stability of the perturbed motion of the "plate-flow" dynamic system (1)-(4) are received.

With the help of graphic-analytical and numerical methods of analysis, the multiparametric space of the "plate-flow" system was partitioned into a stability domain and the instability domain in the form of divergence and flutter.

The concept of "chain of transitions" is introduced, the links of which are transitions from one domain to another. "Chains of transitions" allows you to visually illustrated the dynamics of the behavior of the disturbed motion of the "plate-flow" system. in the space of its parameters.

The critical velocities of a supersonic gas flow are found, when exceeding which the disturbed motion of the dynamic system "plate - flow" loses stability in the forms of a panel divergence and a panel flutter under the assumption that in the plate at the moment " buckling "only bending stresses arise.

For values of parameter $\gamma \ll 1$ is showned, that the effect of the compressive stress on the stability of the system becomes imperceptibly small.

The minimum relative thickness of steel elongated plate determined, at which the perturbed motion of a dynamic system near the beginning of the interval of supersonic velocities is stable. As it turned out, in this case, the reduced critical velocities of the divergence and panel flutter are increasing functions of the stress coefficient. Thus, in the case of elongated plates, the relative thickness of which is less than the minimum, the initial stress state leads to stabilization of the disturbed motion of the system.

\section{REFERENCES}

1. Volmir A.S. The Stability of the elastic system. Moscow: Physmathgiz. 1963. 880 p.

2. Bolotin V.V. Nonconservative Problems of the Theory of Elastic Stability, $340 \mathrm{p}$. Izd. Fizmat lit. Moscow. 
3.. Ishlinskii A.Yu. About the same, limit transition in the theory of stability of elastic rectangular plates. // Reports of USSR Academy of Sciences. 1954. V. 95. No. 3. Pp. 38-46. 4. Movchan A.A.(1956). About vibrations of a plate, moving in gas. Izv. Acad. Nauk USSR. PMM. V. 20. No. 2, pp. 211-222.

5. Volmir A.S. (1972). Nonlinear dynamics of plates and shells. Nauka. Moscow. 432p.

6. Tovstic P.E. Stability of the thin plate: Asymptotic metods // Moscow: Science. Physmathlit. 1995. 320 p.

7. Strength. Stability. Vibrations. Directory in 3 v. // Under the editorship of I. A. Birger and Ya. G. Panovko. - Moscow.: Mechanical Engineering. 1968.

8. Algazin, S.D., Kijko, I.A. (2006). Flutter of Plates and Shells. Nauka. Moscow. 247 p.

9. Novichkov Yu. N. A flutter of plates and shells. Results of science and technology Mechanics of deformable solids. - Moscow: Science. 1978. V. 11. Pp. 67-122.

10. Crowell A.R., McNamara J.J., Miller B.A. (2011). Hypersonic aerothermoelastic response prediction of skin panels using computational fluid dynamic surrogates. Journal of aeroelasticity and structural dynamics. V. 2. No. 2, p. 3-30.

11. Rzhanitsyn A.R. (1985). "A cantilever elastic beam loaded by a follower force", Izv. Acad. Nauk Arm. SSR, Mekhanika, 38, No. 5, pp. 33-44.

12. Bautin N.N. The behavior of dynamical systems near the boundaries of the stability region. - M.: Science. 1984. 176 p.

13. Il'yushin A.A.(1956). Law of Flat Sections at the Big Supersonic Velocity. PMM, v. 20(6), pp. 733-755.

14. Ashley G H., Zartarian G. Piston theory - a new aerodynamic tool for the aeroelastician // J.Aeronaut. Sci. 1956. Vol. 23. N12. P. 1109-1118.

15. Belubekyan M.V., Martirosyan S.R. On the problem of the flutter of an elastic rectangular plate, when the supersonic gas flow is in a direction perpendicular to the free edge. //Proceed. of NAS of Armenia. Mechanics. 2014. V. 67(2). P. 12-42.

16. Belubekyan M.V., Martirosyan S.R. On a problem of supersonic panel flutter in the presence of concentrated inertial masses and moments // Proceed. of NAS of Armenia. Mechanics. 2016. V. 69(3). P. 41-59.

17. M.V. Belubekyan, S.R. Martirosyan. On divergence of compressed panel in supersonic gas flow, an accumulating on its free edge// Proceed. of NAS of Armenia. Mechanics. 2017. V. 70(4). P.12-34.

\section{Сведения об авторах:}

Белубекян Мелс Вагаршакович - кандидат физ.-мат. наук, профессор, главный научный сотрудник Института механики НАН Армении, Ереван, Армения (+374 10) 521503, (+374 10) 580096

E-mail: mbelubekyan@yahoo.com

Мартиросян Стелла Размиковна - кандидат физ.мат. наук, ведущий научный сотрудник Института механики НАН Армении, Ереван, Армения (+374 10) 524890

E-mail: $\underline{\text { mechinsstella@mail.ru }}$ 